\title{
Origami building blocks: Generic and special four-vertices
}

\author{
Scott Waitukaitis and Martin van Hecke \\ Huygens-Kamerlingh Onnes Lab, Leiden University, P. O. Box 9504, 2300 RA Leiden, The Netherlands \\ and FOM Institute AMOLF, Science Park 104, 1098 XG Amsterdam, The Netherlands
}

(Received 30 July 2015; published 3 February 2016)

\begin{abstract}
Four rigid panels connected by hinges that meet at a point form a four-vertex, the fundamental building block of origami metamaterials. Most materials designed so far are based on the same four-vertex geometry, and little is known regarding how different geometries affect folding behavior. Here we systematically categorize and analyze the geometries and resulting folding motions of Euclidean four-vertices. Comparing the relative sizes of sector angles, we identify three types of generic vertices and two accompanying subtypes. We determine which folds can fully close and the possible mountain-valley assignments. Next, we consider what occurs when sector angles or sums thereof are set equal, which results in 16 special vertex types. One of these, flat-foldable vertices, has been studied extensively, but we show that a wide variety of qualitatively different folding motions exist for the other 15 special and 3 generic types. Our work establishes a straightforward set of rules for understanding the folding motion of both generic and special four-vertices and serves as a roadmap for designing origami metamaterials.
\end{abstract}

DOI: 10.1103/PhysRevE.93.023003

\section{INTRODUCTION}

In recent years physicists, mathematicians, artists, and engineers have shown that origami provides a robust design platform in realms as diverse as robotics [1,2], medical devices [3], battery optimization [4], and mechanical metamaterials [5-18]. In regard to the last category, Schenk and Guest [5] illustrated how the Miura-ori fold tessellation, a pattern originally designed for compacting space cargo [19], is in fact an auxetic two-dimensional (2D) metamaterial. Concurrently, Wei et al. confirmed this result and also connected the geometry of the Miura-ori to the physics by dressing the folds with torsional springs and revealing nonlinear rigidity and bending response [6]. Silverberg et al., again using Miura-ori, discovered that its stiffness is tunable with the introduction of "pop-through" defects [7], while Evans et al. recast tessellated origami into the language of conventional lattice mechanics [10]. Using a more generalized pattern, we showed how mechanical frustration of origami can lead to multistable metamaterials [9].

Though origami has been studied in a wide variety of physical contexts, the common thread is the intimate connection between the physics and the underlying geometry of the fold network. The negative Poisson's ratio behavior observed in Miura-ori, for example, is a purely geometrical consequence of the base folding unit of the tessellation [5,19]. As Wei et al. showed, the same is true for the out-of-plane bending response [6]. Silverberg et al. demonstrated how bistability can arise from "hidden" bending degrees of freedom that separate two low-energy, geometrically isomorphic states [13]. As shown recently, even the lattice mechanics and long-wavelength behavior of origami networks depend explicitly on geometric expansions of the folding motions near the equilibrium state [10]. In our own work, we showed how multistability in fourvertices and four-vertex tessellations is a direct consequence of nonlinearity in the folding motion as a consequence of vertex geometry [9]. These examples make it clear that a better understanding of origami geometries and folding motions is an essential step towards creating new functionalities.
To make progress with this goal in mind, we focus our efforts here on a small, but useful, subset of the space of possible origami geometries. In all of the cited origami metamaterials, the mechanics is governed by one fundamental unit-the four-vertex. This is a structure where four rigid, wedge-shaped plates (with sector angles $\alpha_{i}$ ) surrounded by four folds meet at a point-see Fig. 1(a). The four-vertex is the simplest rigid-plated unit one can consider because it has just one continuous degree of freedom-vertices with fewer folds are fixed. Historically, the focus has been on flat-foldability, i.e., the situation where all folds can close simultaneously. In four-vertices, this is possible when the sum of alternating sector angles is equal $\left(\alpha_{1}+\alpha_{3}=\alpha_{2}+\alpha_{4}\right)$ [20-22]. Most studies thus far have focused on one particular type of four-vertex, the Miura-ori, which, in addition to being flat-foldable, is also highly symmetric [19]. A large space of four-vertex geometry remains to be explored.

We answer many fundamental but largely unaddressed questions: How can four-vertex geometries be categorized? What are the possible mountain-valley arrangements? Which folds can fully close? How many ways can the vertex be folded? What roles do symmetries play? Similarly to what has been done for flat-foldable vertices, we show that much progress can be made by considering maximally folded states and that the answers to these questions can be expressed via relationships between the sector angles. First, we focus on generic four-vertices, i.e., those that have no special relationships (e.g., involving equality signs) between the sector angles, where we identify three distinct types. We establish simple rules that determine the possible mountain-valley arrangements and the folds that can fully close for each type. Moreover, we show that for each type there are two possible subtypes with qualitatively different folding behavior.

Next we move on to special four-vertices, where we allow pairs of sector angles or sums of pairs to be equal. Flat-foldable vertices arise naturally in this scheme, but we show that they are just 1 of 16 distinct special types. These can be distinguished based on their codimensionality within the space of sector angles: nine codimension- 1 vertices, six 
(a)

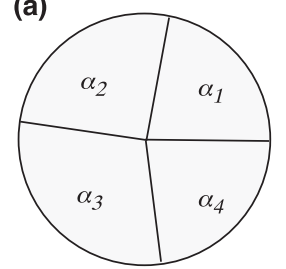

(b)

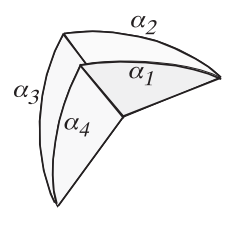

(c)

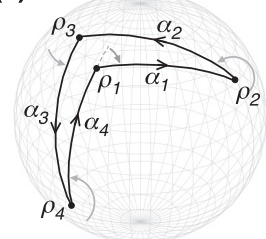

FIG. 1. Geometry of a four-vertex. (a) Illustration of flat fourvertex geometry with sector angles $\alpha_{i}$. (b) Rendering in 3D of vertex from (a) in a partially folded state. (c) The vertex edges make a polygon on the unit sphere, where we use the vertex orientation (arrows) to define the folding angles $\rho_{i}$ as the out-of-plane deviation angle from one plate to the next.

codimension- 2 vertices, and, finally, one codimension-3 vertex in which all sector angles are equal. These special types partition the space of generic four-vertices, dividing it into regions of differing generic types and subtypes. Finally, we show that eight of these have fundamentally different folding branches than generic 4four-vertices [9].

As we discuss and analyze the different generic and special vertex types, we invite the reader to fold example four-vertices from paper (available as cutouts in the Supplemental Material [23]) to illustrate how subtle differences in the flat geometry lead to different folding motions.

\section{GENERIC FOUR-VERTICES}

As shown in Fig. 1(a), a four-vertex consists of four rigid plates with sector angles $\alpha_{i}(i=1,2,3,4$, counted counterclockwise) connected by four folds (or hinges). We assume that $\sum \alpha_{i}=2 \pi$ and that each sector angle is smaller than the sum of the other three $\left(\alpha_{j}<\sum_{i \neq j} \alpha_{i}\right.$-otherwise the vertex folds trivially or is rigid [24]). Figure 1(b) shows a vertex in a partially folded state. Tracing the edges of the vertex, we see that the folded state is equivalent to an oriented, nonintersecting four-polygon on the unit sphere [25]. The foldedness of the vertex is captured by the four folding angles $\rho_{i}$, defined as the deviation from in plane alignment between folds $i-1$ and $i$. For example, two aligned plates have a folding angle $\rho=0$ between them, a counterclockwise turn from one plate to the next (e.g., folds $\rho_{2}, \rho_{3}$, and $\rho_{4}$ in the figure) has a folding angle $\rho>0$, and a clockwise turn has $\rho<0$ (e.g., fold $\rho_{1}$ in the figure).

Generic four-vertices are those for which no special relationships exist between the sector angles. Consequently, there exists a well-defined ordering of the sector angles $a<$ $b<c<d$ [for the example in Fig. 1(a), $\alpha_{1}=a<\alpha_{4}=b<$ $\left.\alpha_{2}=c<\alpha_{3}=d\right]$. The distinct types of generic four-vertices correspond to different arrangements of these ordered angles around the vertex center, which we can represent schematically by placing the letters $a$ through $d$ in four quadrants of a circle, as in Fig. 2. At first glance it appears there are $4 !=24$ such arrangements, but this is reduced when one considers inherent symmetries. We account for discrete rotational symmetry by putting the smallest angle $a$ in the upper right quadrant- this reduces from 24 to 6 . Second, we account for the symmetry associated with flipping the vertex over by choosing the angle in the upper left quadrant to be larger than the one in the

(a)
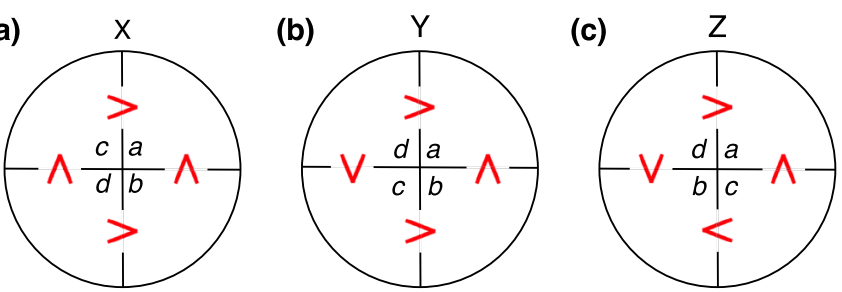

FIG. 2. Generic four-vertices. The three types of generic vertices, $\mathrm{X}, \mathrm{Y}$, and $\mathrm{Z}$, are determined by the arrangement of the ordered sector angles, $a<b<c<d$, around the vertex center. In addition to the letters, the less-than and greater-than signs also indicate the relative sizes of adjacent sector angles.

lower right quadrant. This leaves just three types of generic vertices that cannot be mapped onto each other by reflection or rotations. We call these types X, Y, and Z, as shown in Fig. 2.

\section{A. Generic folding behavior}

Given a particular generic four-vertex, how can we expect it to fold? By introducing the notions of the unique and binding folds, the unique and binding plates, and the dominant pair, the possible folding motions of generic four-vertices can be deduced conveniently. This leads to the conclusions that generic four-vertices have two branches of folding motion and that each generic type can be further categorized into two distinct subtypes. The reader is encouraged to verify and visualize our results by folding the generic vertices included in the Supplemental Material [23].

\section{Unique folds}

It was first shown by Huffman that a Euclidean four-vertex in a partially folded configuration will always have exactly one fold whose folding angle is opposite in sign from the rest [25]. In any other scenario, the vertex must have some net Gaussian curvature-which defies our assumption of a Euclidean vertex. We call folds that are capable of having the opposite sign unique folds. How do we determine which folds are unique?

Suppose for a given vertex folded in some configuration that fold $i$ has the opposite sign-this requires that folds $i+1$, $i+2$, and $i-1$ have the same sign. As shown in Fig. 3(a), (a)

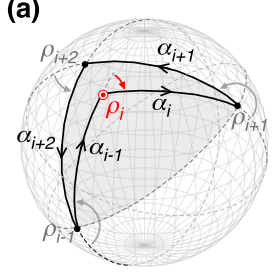

(b)

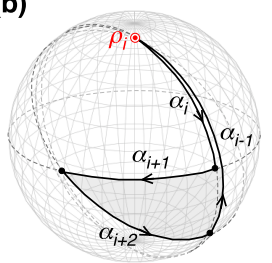

(c)

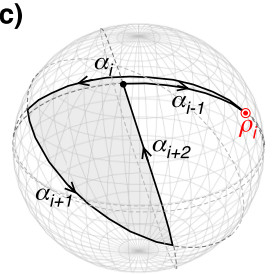

FIG. 3. Unique and binding folds. (a) If fold $i$ is unique in a given configuration, its folding angle must be opposite those of the other folds (e.g., clockwise vs counterclockwise, as shown). This requires that the point corresponding to fold $i$ on the unit sphere lies in the spherical triangle created by the remaining nonunique folds (shaded in the figure). (b) If fold $i$ binds and $\alpha_{i}<\alpha_{i-1}$, then the bound vertex creates a spherical triangle (shaded region in figure) with sides $\left\{\alpha_{i-1}-\alpha_{i}, \alpha_{i+1}, \alpha_{i+2}\right\}$. (c) Otherwise, if $\alpha_{i}>\alpha_{i-1}$, the bound side lengths are $\left\{\alpha_{i}-\alpha_{i-1}, \alpha_{i+1}, \alpha_{i+2}\right\}$. 
for example, these three folds have positive folding angles (counterclockwise turns from one plate to the next). In order for fold $i$ to have the opposite sign, it must lie at some point on the sphere such that it has a negative (clockwise) folding angle. By considering the hemispheres created by the great circles connecting points $i+1 \rightarrow i+2$ and $i+2 \rightarrow i-1$, the location of the point $i$ can be constrained to lie between the minor bigon created by these lines-points outside this region would require either $\rho_{i+1}$ or $\rho_{i-1}$ have the opposite sign from $\rho_{i+2}$. Considering the great arc connecting points $i+$ $1 \rightarrow i-1$, which disects the bigon, we cannot have $i$ on the hemisphere opposite the point $i+2$ because it would give fold $i$ the same sign as the rest of the folds. We therefore conclude that the unique fold must always lie somewhere inside the spherical triangle created by the nonunique folds, as shown in the shaded region in Fig. 3(a). This reveals that if fold $i$ is a unique fold, then it will always satisfy the inequality

$$
\alpha_{i-1}+\alpha_{i}<\alpha_{i+1}+\alpha_{i+2} .
$$

The converse is also true-if a set of sector angles satisfies Eq. (1), then we can construct a vertex where fold $i$ is a unique fold (simply by drawing a figure similar to the one above). It follows that if fold $i$ is a unique fold, then the opposing fold $i+2$ is not and vice versa. Moreover, this means that either fold $i+1$ or fold $i-1$ (but not both) is also a unique fold. Hence, a generic four-vertex will always have two unique folds, which we designate $u$ and $u^{\prime}$, that straddle a common plate [26]. We call this the unique plate and designate it by the letter $U$. For example, for the vertex shown in Fig. 1(a) folds 1 and 2 are the unique folds and plate 1 is the unique plate.

The existence of two unique folds in any generic fourvertex may seem perplexing at first: How can two folds have the opposite sign at the same time? By definition, of course, they cannot, and this is why we have defined a unique fold as one capable of having the opposite sign from the rest. The existence of two unique folds means that four-vertices generically have two branches of folding motion [9]. Each branch of folding motion has a corresponding unique whose sign is opposite from the other folds on that branch. As we will show later, this changes for certain special vertices.

\section{Binding folds}

An observation readily made while folding a generic fourvertex is that some folds are capable of fully closing to $\pm \pi$ while others are not. We call a fold that is capable of fully closing a binding fold. As shown in Figs. 3(b) and 3(c), closing such a fold creates a spherical triangle. If fold $i$ binds and $\alpha_{i}<$ $\alpha_{i-1}$, then the side lengths of the spherical triangle are $\left\{\alpha_{i-1}-\right.$ $\left.\alpha_{i}, \alpha_{i+1}, \alpha_{i+2}\right\}$. Otherwise, if $\alpha_{i}>\alpha_{i-1}$, then they are $\left\{\alpha_{i}-\right.$ $\left.\alpha_{i-1}, \alpha_{i+1}, \alpha_{i+2}\right\}$. In either case, the three sides must obey the three permutations of the spherical triangle inequality, which for generic vertices take the form of strict inequalities, i.e.,

$$
\begin{array}{r}
\alpha_{i}<\alpha_{i-1}:\left\{\begin{aligned}
\alpha_{i-1}-\alpha_{i} & <\alpha_{i+1}+\alpha_{i+2} \\
\alpha_{i+1} & <\alpha_{i+2}+\alpha_{i-1}-\alpha_{i} \\
\alpha_{i+2} & <\alpha_{i-1}-\alpha_{i}+\alpha_{i+1}
\end{aligned}\right. \text { (iii) } \\
\alpha_{i}>\alpha_{i-1}:\left\{\begin{aligned}
\alpha_{i}-\alpha_{i-1} & <\alpha_{i+1}+\alpha_{i+2} \\
\alpha_{i+2} & <\alpha_{i}-\alpha_{i-1}+\alpha_{i+1} \\
\alpha_{i+1} & <\alpha_{i+2}+\alpha_{i}-\alpha_{i-1}
\end{aligned}\right.
\end{array}
$$

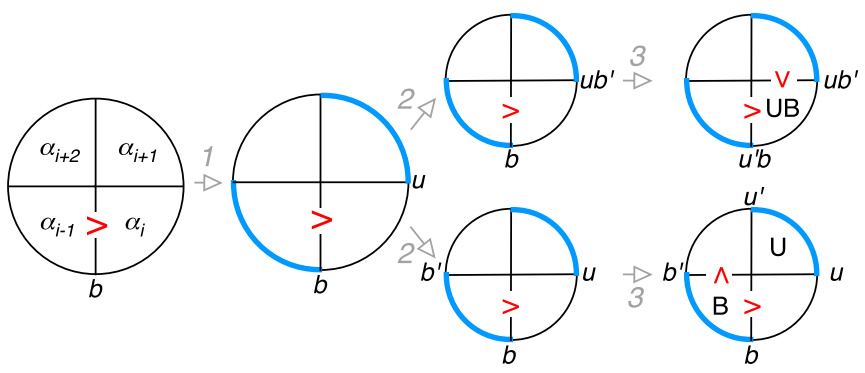

FIG. 4. Classification of vertices by the relative location of the unique and binding plates. We assume that fold $i$ is binding and that $\alpha_{i-1}>\alpha_{i}$. It follows from Eqs. (2) and (3) that (1) fold $i+1$ is unique, and plates $i+1$ and $i-1$ are the dominant pair (indicated by the thick blue rim), and (2) there are two possibilities for the $b^{\prime}$ folds. Once the location of $b^{\prime}$ is determined, (3) the locations of the $u^{\prime}$ fold and the $U$ and $B$ plates are fixed according to Eqs. (2) and (3); this leads to two subtypes with either $U$ and $B$ the same (top) or opposite (bottom).

Conversely, if a set of sector angles obeys one of the sets of inequalities above, then an arrangement can be draw on the unit sphere such that fold $i$ is equal to $\pm \pi$. As with the unique folds, these inequalities imply that generic vertices have two binding folds (denoted $b$ and $b^{\prime}$ ) that straddle a common plate (denoted $B$ ). Looking specifically at Eqs. 2(ii) and 3(ii), we see that if fold $i$ is binding, then either $i+1$ or $i-1$ is unique. This is consistent with Fig. 3, where we see that, on a particular branch, the binding fold always has its corresponding unique fold next to it.

\section{The dominant pair and generic subtypes}

Equations 2(iii) and 3(iii) involve the two pairs of opposing plates, specifically addressing for which pair the sum of the sector angles is larger (and thus larger than $\pi$ ). We call this pair the dominant pair, and we can use it to quickly identify the unique and binding plates of a generic vertex. Figure 4 illustrates that the connection between unique and binding folds can be reformulated as follows: If fold $i$ is a binding fold, then the neighboring fold separated by the smaller of the neighboring plates is the corresponding unique fold, $u$, and the inequalities expressed in Eqs. 2(iii) and 3(iii) lead to a definite arrangement of the dominant pair. By then considering the two possible locations for the other binding fold, $b^{\prime}$, we can easily establish two different possible folded configurations for plate $U$, plate $B$, and the dominant pair (Fig. 4). It follows by inspection that the sector angle for plate $B$ is a local extremum. If the $B$ plate is a local minimum, then the $U$ and $B$ plates are equal, and the $U$ plate is not part of the dominant pair. If plate $B$ is a local maximum, then the $U$ and $B$ plates are opposite and the $U$ plate is part of the dominant pair.

In Fig. 5, we use the rules regarding the dominant pair to show that for each generic type there are two possible arrangements of the unique and binding folds on the first branch $(u$ and $b)$ and on the second branch $\left(u^{\prime}\right.$ and $\left.b^{\prime}\right)$, This reveals that generic vertices come in two subtypes depending on the relative locations of $U$ and $B$, which we will call subtype 1 when they are the same (here the $B$ plate is always a local minimum) and subtype 2 when they are opposite (here the $B$ plate is always a local maximum). 
(a)

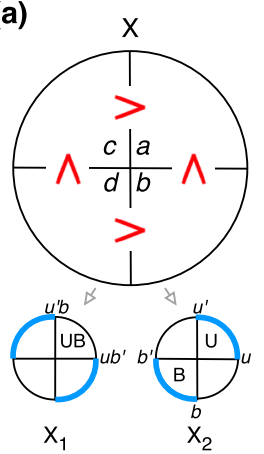

(b)

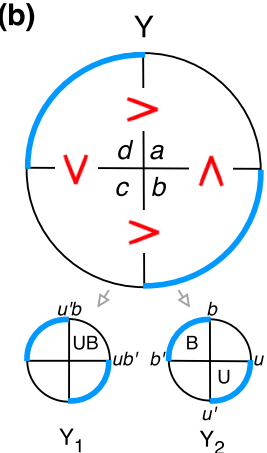

(c)

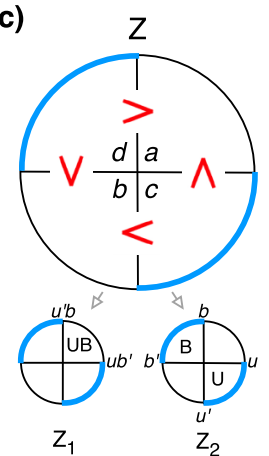

FIG. 5. Subtypes and folding branches for generic vertices. For each type and subtype, the dominant pair is indicated by the thick blue rim and the unique and binding plates by $U$ and $B$, respectively. The folding branches on each subtype correspond either to folds $u$ being unique and $b$ binding or $u^{\prime}$ being unique and $b^{\prime}$ binding.

We note in passing that the subtype classification is closely related to the Grashof classification of (spherical) four-bar linkages. A Grashof linkage is one where (at least) one of the output bars (equivalent to plates) can rotate continuously, which is useful for performing engineering tasks. This is possible if the sum of the shortest and longest bars is greater than the sum of the remaining two. This translates to $a+d<$ $b+c$, and for all three generic vertices this is precisely the condition for subtype 1 . Hence, even though spherical linkages and origami differ in that (a) the vertices here have sector angles that add to $2 \pi$ and (b) cannot self-intersect [27], subtype 1 vertices are related to (nonintersecting) Grashof linkages $[28,29]$.

\section{SPECIAL FOUR-VERTICES}

Special vertices arise when sector angles or sums of pairs of sector angles are equal. Incorporating such constraints changes the combinatorics in determining the possible types of vertices, ultimately resulting in 16 new ones. Furthermore, this allows for the possibility that some of the inequalities that govern the unique and binding folds become equalities, which changes the nature of the folding motion and, in some cases, the number of folding branches. For example, the well-studied class of flat-foldable four-vertices occurs when there is no dominant pair, i.e., when the sums of even and odd sector angles are equal. In that case, all folds close simultaneously. Clearly, qualitatively different rules apply in vertices such as these. (In principle, one could consider more exotic constraints such as products, etc. However, as can be verified by looking at the vertex folding equations, the motion depends solely on sums and differences. This means that the relevant relationships correspond to individual sector angles or sums thereof being equal $[9,10,25]$.)

In this section, we systematically determine all special vertex types. We subdivide these based on the number of incorporated constraints, resulting in codimension-1, -2 , and -3 special vertices. We show how the relationships among these and the generic vertices can be visualized by looking at regions, lines, and points in 2D subspaces of the full, 3D space of sector angles. Finally, we analyze each special type

and determine the locations of their unique and binding folds and further characterize their folding branches.

\section{A. Codimension-1 vertices}

The simplest of the codimension-1 special vertices occurs when two sector angles are equal. This disrupts the welldefined ordering of generic vertices in one of three ways: the equal angles can be the smallest $(a=b)$, the middle $(b=c)$, or the largest $(c=d)$. For each of these possibilities, the equal angles can be arranged next to each other or opposite to each other. This makes it clear that six special vertex types are associated with two angles being equal, as in Fig. 6(a). We give these the names 2NS, 2NL, 2NM, 2OS, 2OL, and 2OM, where the " 2 " stands for two angles being equal, "N" and "O" distinguishe whether the equal angles are arranged next to or opposite each other, and "S," "L," and "M" identify them as the smallest, largest or middle. Like generic vertices, our notions of the $U$ plate, the $\bar{B}$ plate, and the dominant pair remain well defined for these vertices. Unlike generic vertices, however, only $2 \mathrm{NM}$ and $2 \mathrm{OM}$ vertices have two subtypes, whereas for all other special vertices the subtype is fixed via the constraints.

Setting the sums of pairs of angles equal results in more codimension-1 special types. Like generic vertices, these still have a well-defined ordering of the sector angles $a<b<c<$ $d$. The equal sums must therefore be $a+d=b+c=\pi$. (In the language of spherical linkages these would be classified as change point mechanisms [29]). The preserved ordering signifies that these come in three varieties that are derived directly from each of the generic types. One of these is the familiar flat-foldable vertices, which we denote GFF as they are the most general ones possible. Interestingly, GFF vertices are only derived from generic type $\mathrm{X}$. The other two types have the angles that sum to $\pi$ arranged next to each other, which results in two opposing folds lying on precisely the same line. We give these the names CY and CZ, with "C" denoting that they have collinear folds and "Y" and " $Z$ " signifying the parent generic type. As with most of the special types generated from setting two angles equal, the additional constraints here preclude distinct subtypes. We will show in the next section (and in particular Fig. 8) that these vertices require modifications to our notions of the $U$ and $B$ plates.

\section{B. Codimension-2 and codimension-3 vertices}

Adding another constraint results in codimension-2 vertices, and the simplest of these occurs when three sector angles are equal. We can quickly determine that there are just two of these by inserting two equalities into our ordered angles, i.e., $a=b=c<d$ or $a<b=c=d$. We designate these 3S and $3 \mathrm{~L}$. As with vertices where two angles are equal, these still have a well-defined dominant pair and $U$ and $B$ plates.

Codimension-2 vertices can also arise via the combination of two equal angles and equal sums of pairs of angles; this results in four types. In two of these, the angles that are equal also add to $\pi$-hence they are $\pi / 2$. They can be arranged next to each other, which results in another collinear vertex (2NC), or they can be arranged opposite each other, which results in a subclass of flat-foldable vertices (2OFF). Alternatively, if the equal angles do not add to $\pi$, then the result is a double 
(a)
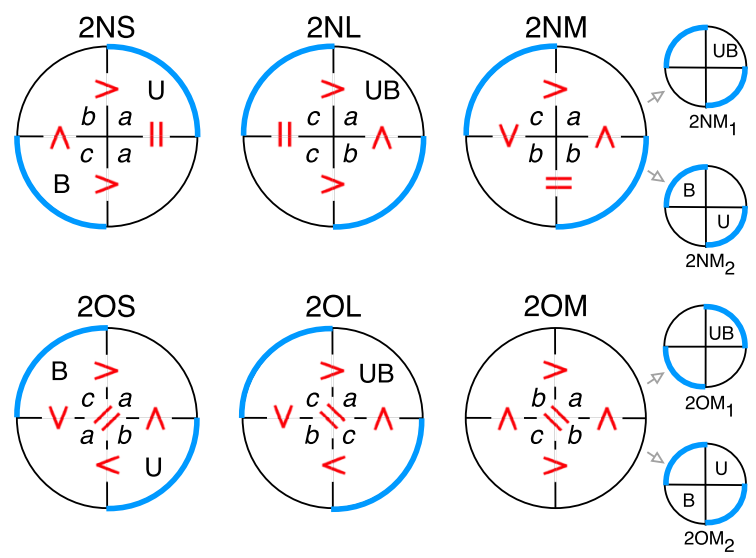

(b)
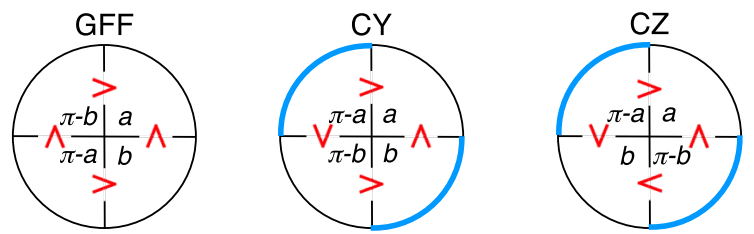

(c)

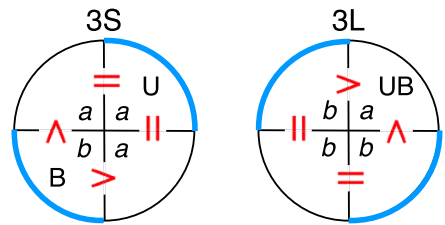

(d)

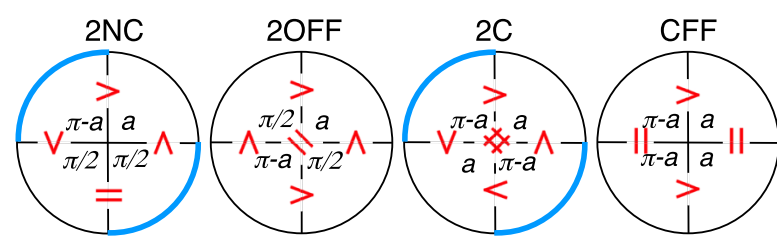

(e)

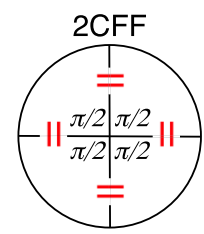

FIG. 6. Special four-vertices. Six codimension-1 special types arise if two sector angles are equal (a). Three more arise when sums of pairs are equal (b). Codimension-2 vertices include ones with three sector angles equal (c) and also those with two equal and sums of pairs equal (d). The only codimension- 3 vertex has all sector angles equal to $\pi / 2$ (e). The relative sizes are indicated both by letters $(a<b<c<d)$ and also by equal and less-than and greater-than signs. In (a) and (c), the unique and binding plates are well defined (as indicated), but in all other types this is not the case-we discuss this more fully in Fig. 8. For all vertices except 2NM and 2OM, the subtype is predetermined by the constraints.

collinear vertex, $2 \mathrm{C}$, or a collinear vertex that is also flatfoldable, CFF. This last type, CFF, is the base vertex for the Miura-ori. (In the midst of the wide variety of other vertices we have identified, it should be clear that this type occupies a very small region of phase space.) Adding any additional constraints results in the singular codimension- 3 vertex, $2 \mathrm{CFF}$, where all sector angles are equal to $\pi / 2$.

\section{Visualizing the full geometric space}

The schematics in Figs. 2 and 6 are useful for identifying vertex types, but to see the connections between different types we now more carefully consider the configuration space spanned by the $\alpha_{i}$. As the sum of sector angles equals $2 \pi$, generic Euclidean vertices occupy the bulk of a 3D space. We can specify a point in this space using $\alpha_{1,2,3}$ as coordinates $\left(\alpha_{4}:=2 \pi-\sum_{i=1,3} \alpha_{i}\right)$. The special vertices reside on nine distinct planes (codimension-1), six lines (codimension-2), and one point (codimension-3).

While we cannot easily depict this 3D space, we gain insight by looking at $2 \mathrm{D}$ slices corresponding to fixing one sector angle, as presented in Fig. 7. (For a video traversing the full 3D space, see the Supplemental Material [23].) We see the layout of generic vertices by coloring each region according to type and shading by subtype. The codimension- 1 special vertices, which occupy planes in the full space but lines in this 2D space, are now seen to create divisions between generic types and subtypes. For example, the boundaries between generic types are delineated by four lines $n_{i j}$, corresponding to adjacent folds $i$ and $j$ being equal. The different generic subtypes are bounded by the lines $f f, c_{12}$, and $c_{23}$, which correspond to the flatfoldable vertices $\left(\alpha_{1}+\alpha_{3}=\alpha_{2}+\alpha_{4}\right)$, and the two possible arrangements for collinear folds $\left(\alpha_{i}+\alpha_{j}=\pi\right.$ for $\left.c_{i j}\right)$.

In the lower panels, we connect this to the 16 special types as previously identified. The lines $n_{i j}$ and $o_{i j}$ are split into the special types where two angles are equal, while the lines $f f$ and $c_{i j}$ are split into the special types corresponding to equal sums. The intersection points of lines corresponding to codimension- 2 and codimension-3 vertices bring to light particularly interesting features. For example, $3 \mathrm{~S}$ and $3 \mathrm{~L}$ vertices live at the intersections of different $n_{i j}$ and $o_{i j}$ lines, and any of the three generic types can be reached via an infinitesimal deviation away from these highly constrained domains. As another example, the plots make it readily apparent that $2 \mathrm{C}$ vertices live only in generic type $\mathrm{Z}$. These relationships and more are summarized in Table I, which highlights several other interdependencies: For example, that flat-foldable vertices $(f f)$ that have two equal neighboring plates $\left(n_{i j}\right)$ are automatically collinear $\left(c_{i j}\right)$.

\section{Special folding behavior}

We now analyze the folding behavior for special vertices. For types where two angles are equal [Fig. 6(a)] or three angles are equal [Fig. 6(c)], the dominant pair and $U$ and $B$ plates are well defined. This implies the folding behavior of the two branches can be deduced in the same way as for generic vertices. All of the other special types, however, involve either flat-foldability, collinearity, or a combination thereof, and this creates substantive changes in their folding behavior. We now consider the specific modifications that arise in each of these cases. In Fig. 9, we take these modifications into account and sketch out all folding branches for each of the affected special types.

\section{Flat-foldable vertices}

Flat-foldable vertices are, by definition, those where all folds are binding folds. This becomes apparent in the maximally folded state, illustrated for example in Fig. 8(a), where 

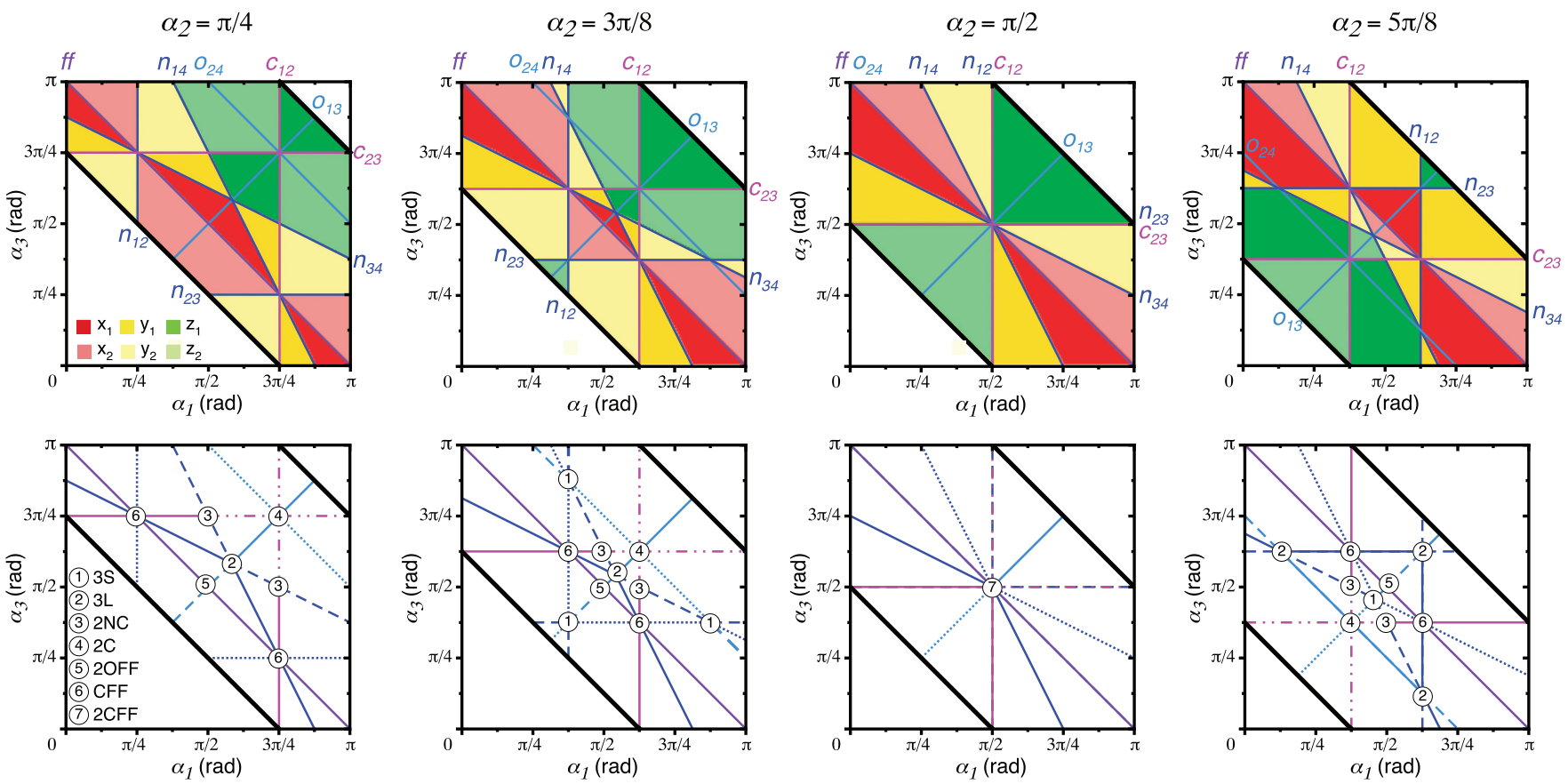

FIG. 7. Visualizing the full space of four-vertices. The top row shows planes of constant $\alpha_{2}$ (values indicated above). White areas are forbidden as one sector angle becomes $<0$ or $>\pi$. The colors denote the generic type (red $=\mathrm{X}$, yellow $=\mathrm{Y}$, green $=\mathrm{Z}$ ) and subtypes (darker $=1$, lighter $=2$ ). Nine lines partition the generic types and subtypes: $n_{i j}$ where neighboring sector angles $\alpha_{i}=\alpha_{j}($ four dark blue lines); $o_{i j}$ where opposite sector angles $\alpha_{i}=\alpha_{j}$ (2 light blue lines); $c_{i j}$, or collinear lines, where the sum of the neighboring sector angles $\alpha_{i}+\alpha_{j}=\pi$ (2 pink lines); and $f f$ where $\alpha_{1}+\alpha_{3}=\alpha_{2}+\alpha_{4}=\pi$ (purple line). The bottom row recasts this based on the codimension-1, -2 , and -3 special vertex types. Lines (codimension-1) are colored as before, but here dots correspond to smallest angles equal, dashes to middle angles equal, and solid lines to largest angles equal. The flat-foldable line is unchanged, but special types CY and CZ now have solid and dash-dot-dot lines, respectively. The line intersections corresponding to codimension- 2 and -3 vertices are indicated by the legend in the leftmost panel. For an animation showing the full 3D space of slices, see the supplemental video [23].

we draw a nearly closed GFF vertex. Flat-foldability alone does not change what we have previously said regarding the unique plate-this is because the angles that add to $\pi$ are opposite from each other. For generic flat-foldable (GFF) (a)

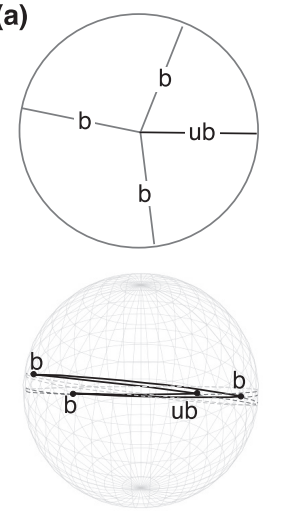

(b)

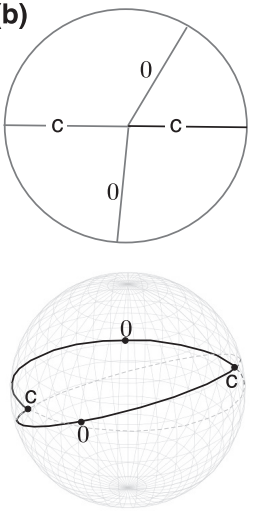

(c)

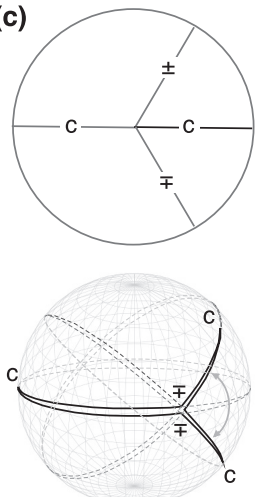

FIG. 8. Special folding motions. (a) Two-dimensional sketch showing that in flat-foldable vertices, all four folds are binding and unique folds remain well defined. (b) For collinear vertices, one branch of folding motion corresponds to the two collinear folds (denoted by $c$ ) being equal while the remaining null folds (denoted by 0 ) stay flat. (c) For a vertex with collinearity and flat-foldability, additional folding branches emerge when the collinear folds bind at $\pm \pi$ and the previously null folds fall on top of each other and permit further motion (indicated by the curved arrow in the figure). vertices, as well as for $2 \mathrm{OFF}$ vertices, there is a well-defined unique plate and two branches of folding motion, but all folds are capable of binding—see Fig. 9.

\section{Collinear vertices}

Collinear vertices have two opposite folds aligned, and this modifies the behavior of one of the folding branches.

TABLE I. Connections among special vertices. Each vertical block indicates a different vertex codimension, with the vertex types as indicated. The vertical columns indicate which conditions are met: $n_{i j}$ for equal neighboring angles, $o_{i j}$ for equal opposite angles, $c_{i j}$ for collinear folds, and $f f$ for a flat-foldability.

\begin{tabular}{lcccc}
\hline \hline$n_{i j}$ & $o_{i j}$ & $c_{i j}$ & $f f$ & Type \\
\hline & & & & $\mathrm{X}_{1}, \mathrm{X}_{2}, \mathrm{Y}_{1}, \mathrm{Y}_{2}, \mathrm{Z}_{1}, \mathrm{Z}_{2}$ \\
$\bullet$ & & & $2 \mathrm{NS}, 2 \mathrm{NM}, 2 \mathrm{NL}$ \\
& $\bullet$ & & & $2 \mathrm{OS}, 2 \mathrm{OM}, 2 \mathrm{OL}$ \\
& & $\bullet$ & & $\mathrm{CY}, \mathrm{CZ}$ \\
$\bullet$ & $\bullet$ & & & $\mathrm{GFF}$ \\
$\bullet$ & & $\bullet$ & & $2 \mathrm{~S}, 3 \mathrm{~L}$ \\
& $\bullet$ & & $\bullet$ & $2 \mathrm{NC}$ \\
$\bullet$ & $\bullet$ & $\bullet$ & & $2 \mathrm{CFF}$ \\
$\bullet$ & $\bullet$ & $\bullet$ & $\bullet$ & $\mathrm{CFF}$ \\
\hline \hline
\end{tabular}



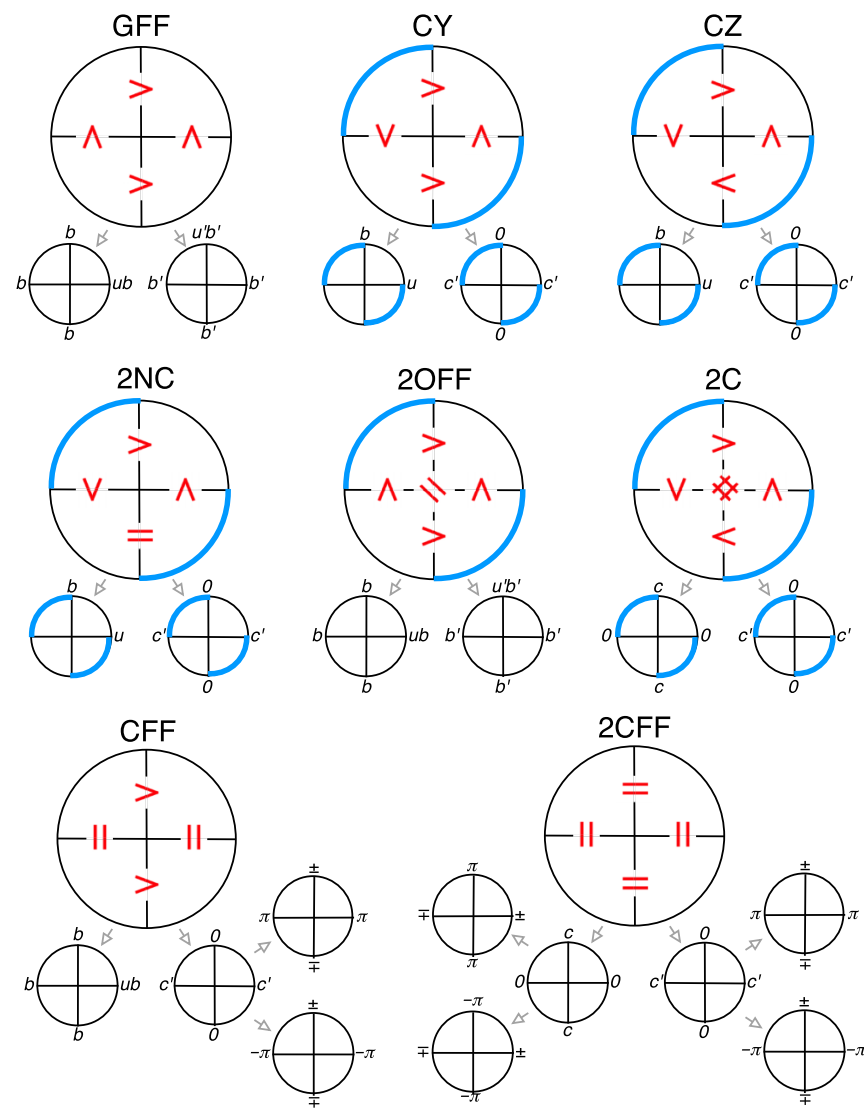

FIG. 9. Vertices with special folding branches. For vertices that are flat-foldable, all folds are capable of binding on all branches-this applies to types GFF, 2OFF, CFF, and 2CFF. For vertices that have collinearity, one branch corresponds to the collinear folds (denoted with a $c$ ) folding simultaneously while the null folds (denoted with a 0) remain flat. Types $\mathrm{CY}, \mathrm{CZ}, 2 \mathrm{NC}$, and $\mathrm{CFF}$ have a single collinearity, while types $2 \mathrm{C}$ and $2 \mathrm{CFF}$ have two. Finally, vertices that have reflection symmetry across collinear folds develop new branches when the collinear folds reach $\pm \pi$. For these branches, the two previously null folds align and can vary continuously between $\pm \pi$ but have opposite signs, here indicated by \pm and $\mp$. We encourage the reader to use the paper cutouts in the supplemental material to illustrate these concepts [23].

As illustrated in Fig. 8(b), collinear vertices have one branch where two of the folds are fixed flat, i.e., they have a value of zero throughout the entire folding motion. We call this the collinear branch, and we distinguish here the collinear folds, which close together, from the null folds, which remain flat. Note that both of the collinear folds will bind during this folding motion. The other branch behaves normally, and thus we conclude that a single collinearity introduces two null folds and two binding folds-see types CY, CZ, and 2NC in Fig. 9. A double collinearity (as in 2C) simply leads to two collinear branches - each of the folds can be binding, and there are no unique folds.

\section{Collinear and flat-foldability}

Finally, consider what happens when there is both flatfoldability and collinearity. This situation results in CFF or $2 \mathrm{CFF}$ vertices with reflection symmetry across the pair of collinear folds (see Table I), as in Fig. 8(c). Once a collinear branch is maximally folded, the previously null folds align on top of each other, and this enables new branches of motion. Here the collinear folds remain fixed at either $\pm \pi$, while the previously null folds are free to vary from $-\pi$ to $\pi$. During this motion these fold angles have equal magnitude but opposite sign; thus in some sense they are unique, but only on these new branches. For type CFF, there is reflection symmetry across a single collinear fold, thus two new branches are introduced, giving rise to four branches in total. For type $2 \mathrm{CFF}$, there is reflection symmetry across both pairs of collinear folds, which means these have six branches of folding motion-see Fig. 9.

\section{SUMMARY AND OUTLOOK}

Motivated by the connection between geometry and mechanical functionality, we have fully characterized the folding motion of rigid Euclidean four-vertices. First, we have shown that there are three generic vertex types, which correspond to the three unique ordered arrangements of the sector angles around the vertex center. Generic vertices have two unique folds that straddle a common plate $U$ and two corresponding binding folds that straddle a common plate $B$, each set corresponding to one of the two unique branches of motion. By drawing connections between the unique folds, binding folds, and dominant pair, we have also shown that generic vertices come in two subtypes depending on whether the $U$ and $B$ plates are the same or opposite to each other.

By introducing constraints between the sector angles, we have shown that there are 16 different types of special vertices. The simplest of these are the codimension- 1 vertices, which occur either when two angles are equal (resulting in six special types) or when sums of pairs of angles are equal (resulting in three special types). Adding one more constraint results in codimension- 2 vertices, which occur when three angles are equal (two types) or when two angles are equal and sums of pairs are equal (four types). Finally, there is just one codimension-3 vertex corresponding to all sector angles equal to $\pi / 2$. For vertices that have flat-foldability, collinearity, or reflection symmetry across folds, the folding motion is dramatically changed - the notions of the binding fold and unique folds must be modified and new branches of folding motion can emerge. In principle, similar organizational schemes could be used to categorize and study vertices with more folds or which are non-Euclidean.

Finally, we would like to stress that the majority of recent work on origami metamaterials has focused on tilings of the codimension-2 CFF vertex, which exhibit very interesting and useful behaviors. Our results highlight the wide range of generic and nongeneric four-vertices, and we suggest that much can be gained by looking at materials made from these other types of vertices.

\section{ACKNOWLEDGMENTS}

We thank C. Coulais, R. Menaut, P. Dieleman, C. Santangelo, and A. Evans for productive discussions and support from NWO via a VICI grant. This work is part of the research programme of the Foundation for Fundamental Research on Matter (FOM), which is part of the Netherlands Organisation for Scientific Research (NWO). 
[1] S. Felton, M. Tolley, E. Demaine, R. Rus, and R. Wood, Science 345, 644 (2014).

[2] R. J. Wood, E. Hawkes, B. K. An, N. M. Benbernou, and H. Tanaka, Proc. Natl. Acad. Sci. U.S.A. 107, 12441 (2010).

[3] K. Kuribayashi et al., Mat. Sci. Eng. A 419, 131 (2006).

[4] Z. Song et al., Nat. Commun. 5, 3140 (2014).

[5] M. Schenk and S. D. Guest, Proc. Natl. Acad. Sci. U.S.A. 110, 3276 (2013).

[6] Z. Y. Wei, Z. V. Guo, L. Dudte, H. Y. Liang, and L. Mahadevan, Phys. Rev. Lett. 110, 215501 (2013).

[7] J. L. Silverberg, A. A. Evans, L. McLeod, R. C. Hayward, T. Hull, C. D. Santangelo, and I. Cohen, Science 345, 647 (2014).

[8] C. Lv, D. Krishnaraju, G. Konjevod, H. Yu, and H. Jiang, Sci. Rep. 4, 5979 (2014).

[9] S. Waitukaitis, R. Ménaut, Bryan Gin-ge Chen, and M. van Hecke, Phys. Rev. Lett. 114, 055503 (2015).

[10] A. A. Evans, J. L. Silverberg, and C. D. Santangelo, Phys. Rev. E 92, 013205 (2015).

[11] H. Yasuda and J. Yang, Phys. Rev. Lett. 114, 185502 (2015).

[12] M. A. Dias, L. H. Dudte, L. Mahadevan, and C. D. Santangelo, Phys. Rev. Lett. 109, 114301 (2012).

[13] J. L. Silverberg et al., Nat. Mater. 14, 389 (2015).

[14] J. Na et al., Adv. Mater. 27, 79 (2015).

[15] K. C. Cheung, T. Tachi, S. Calisch, and K. Miura, Smart Mater. Struct. 23, 094012 (2014).

[16] S. Liua, G. Lua, Y. Chenc, and Y. W. Leongd, Int. J. Mech. Sci. 99, 130 (2015).

[17] F. Lechenault, B. Thiria, and M. Adda-Bedia, Phys. Rev. Lett. 112, 244301 (2014).

[18] B. H. Hanna et al., Smart Mater. Struct. 23, 094009 (2014).
[19] K. Miura, in Proceedings of 31st Congress International Astronautical Federation, Tokyo, 1980, IAF-80-A 31:1-10.

[20] T. Kawasaki, On the relation between mountain-creases and valley-creases of a flat origami, in Proc. 1st Int. Meeting Origami Sci. Tech., edited by H. Huzita (Ferrara, Italy, 1989).

[21] J. Justin, Aspects mathématiques du pliage de papier, in Proc. 1st Int. Meeting Origami Sci. Tech., edited by H. Huzita (Ferrara, Italy, 1989).

[22] E. D. Demaine and J. O'Rourke, Geometric Folding Algorithms: Linkages, Origami, Polyhedra (Cambridge University Press, New York, 2007).

[23] See Supplemental Material at http://link.aps.org/supplemental/ 10.1103/PhysRevE.93.023003 for example paper cutouts for all vertex types and subtypes, and for an animation that illustrates the full 3D geometrical space of Euclidean fourvertices.

[24] If one sector angle is equal to the other three, then it is equal to $\pi$. In this case the vertex has one simple branch of motion where the two aligned folds that straddle this sector angle simply open or close simultaneously. If one angle is larger than the other three, then the vertex is fixed.

[25] D. A. Huffman, IEEE T. Comput. 25, 1010 (1976).

[26] The distinction between nonprimed and primed folds is arbitrary but will become useful when we determine the corresponding binding folds.

[27] s.-m. belcastro and T. Hull, Linear Algebra Appl. 348, 273 (2002).

[28] C. H. Chiang, Mech. Mach. Theory 19, 283 (1984).

[29] C. B. Barker, Mech. Mach. Theory 20, 535 (1985). 


\section{GENERIC VERTICES}
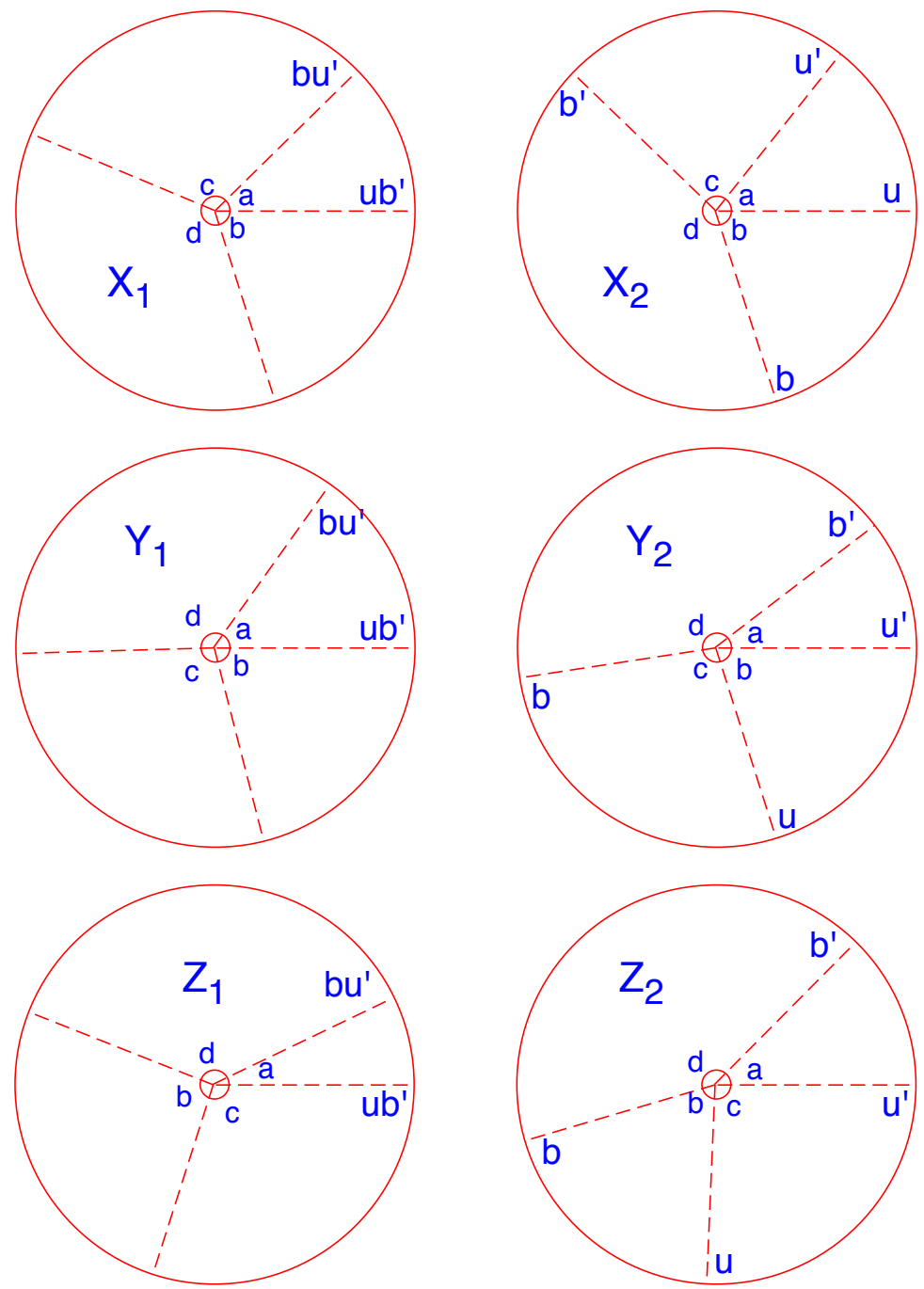


\section{CODIMENSION-1 VERTICES}

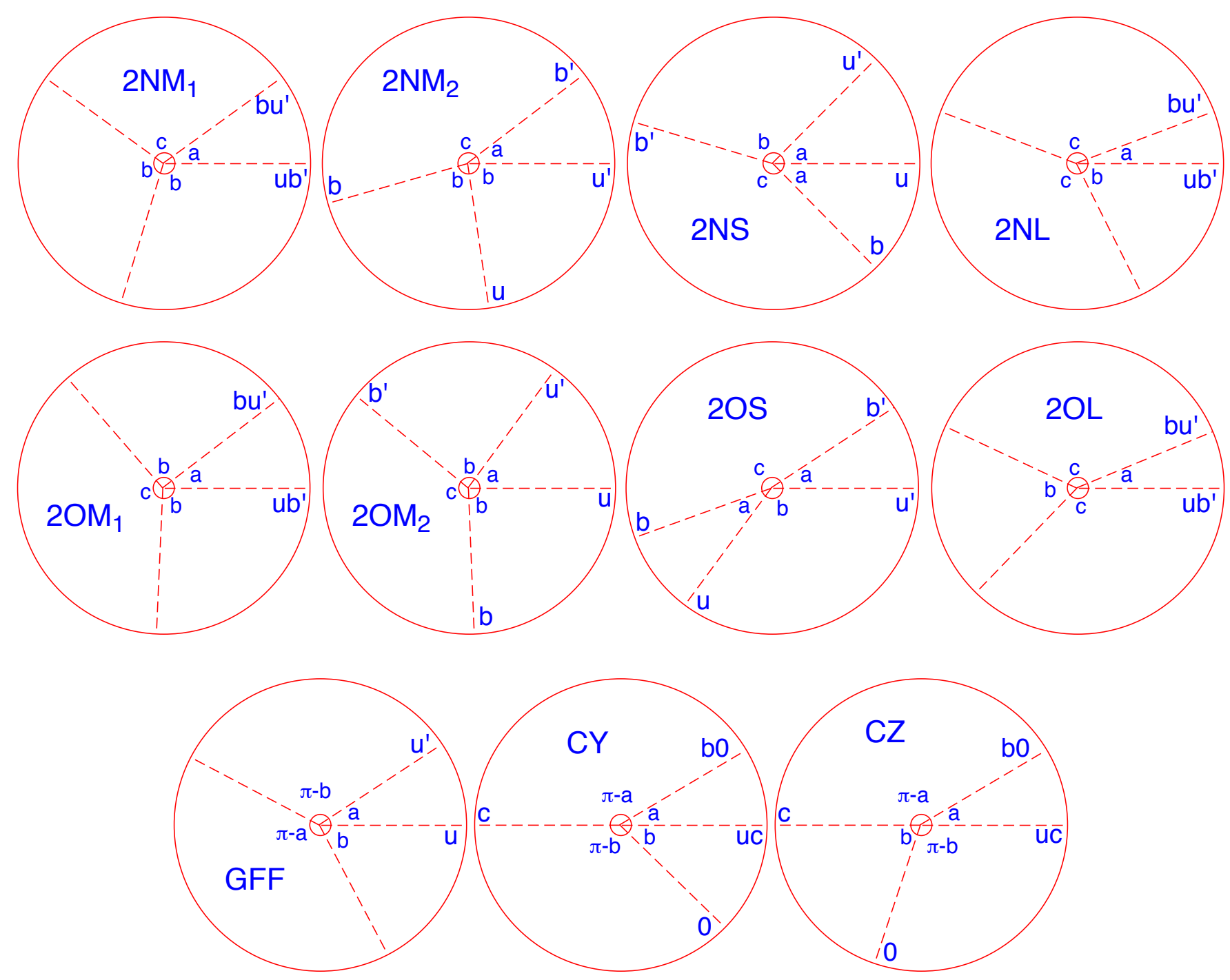




\section{CODIMENSION-2 \& -3 VERTICES}
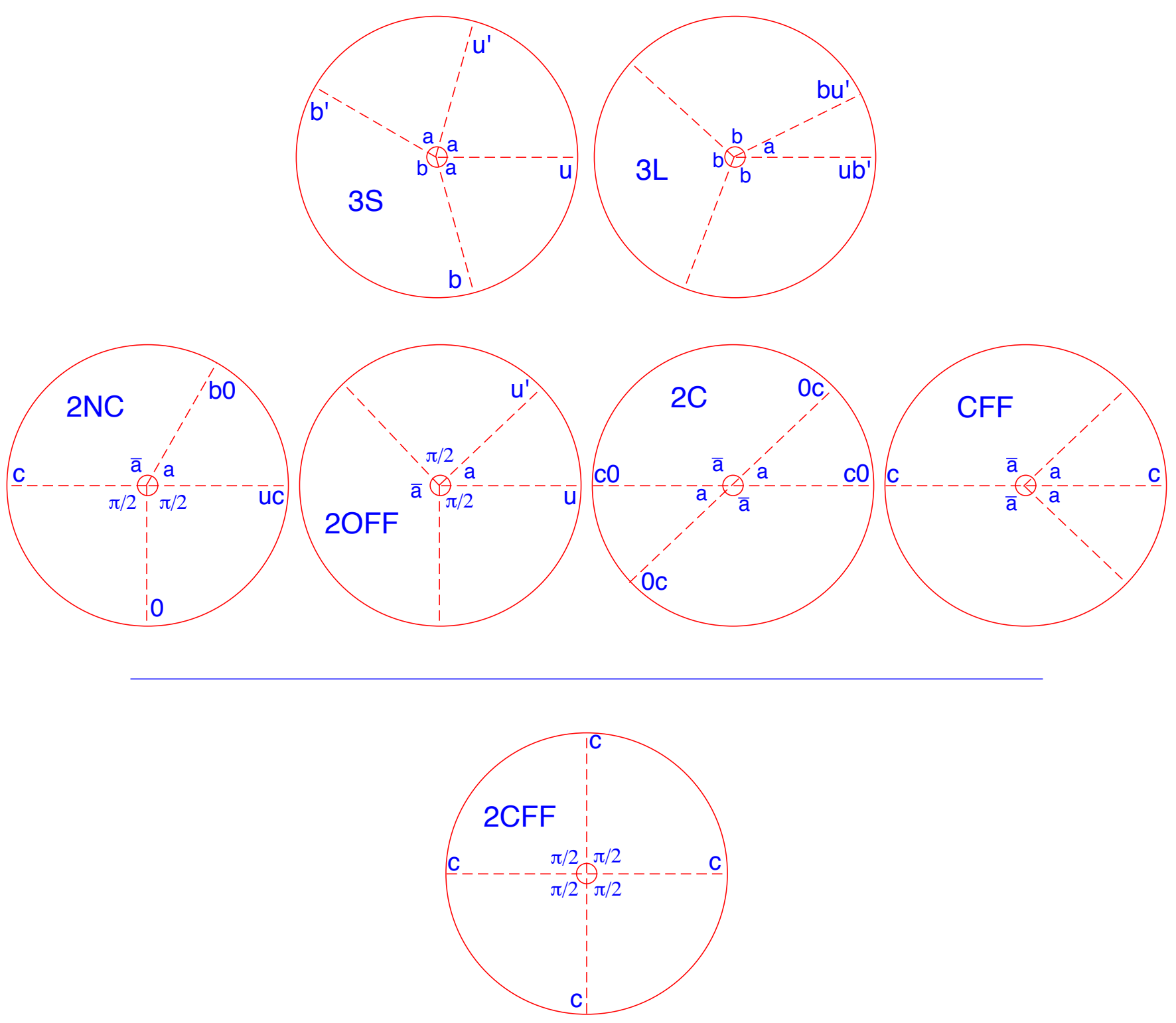